\title{
Editorial: Emerging Trends on Data Analytics at the Network Edge
}

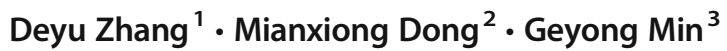

Published online: 24 July 2020

(C) Springer Science+Business Media, LLC, part of Springer Nature 2020

Data analytics at the network edge has received extensive attentions from both the academia and industry due to its potential in various smart applications, such as augmented/ virtual reality, and real-time video surveillance. However, the computational intensiveness and complex full-stack system design have impeded its flourish in our daily life.

This special issue, i.e., "Emerging Trends on Data Analytics at the Network Edge", solicits and publishes original research papers on the algorithms, system design, and methodologies to improve the performance of data analytics performed on the network edge. After a rigorous peer review process, this special issue accepted only 11 papers from 27 submissions. The topics of the accepted paper range from content prefeching to hardware performance measurement. A brief review of the papers included in the special issue is provided as follows.

The first paper, "Efficient Caching Strategy in Wireless Networks with Mobile Edge Computing" by Ying Chen, Shuang Chen, et al. investigates caching in a multi-content provider and multiple users scenario. Aiming at maximizing the caching utility, the authors establish a game model of caching resource competition and prove the existence of Nash equilibrium.

The second paper, "A System for Real-time Intervention in Negative Emotional Contagion in a Smart Classroom Deployed under Edge Computing Service Infrastructure" by

This article is part of the Topical Collection: Special Issue on Emerging Trends on Data Analytics at the Network Edge

Guest Editors: Deyu Zhang, Geyong Min, and Mianxiong Dong

Deyu Zhang

zdy876@csu.edu.cn

Mianxiong Dong

mx.dong@csse.muroran-it.ac.jp

Geyong Min

g.min@exeter.ac.uk

Central South University, Changsha, China

2 Muroran Institute of Technology, Muroran, Japan

3 University of Exeter, Exeter EX4 4PY, UK
Jian Li, Daqian Shi, et al., designs an emotional contagion model for classroom scene to locate the source of negative emotional contagion. They deploy the emotion recognition system as an edge computing-based service to minimize the response time.

The third paper, "Vehicles joint UAVs to Acquire and Analyze Data for Topology Discovery in Large-Scale IoT Systems" by Haojun Teng, Kaoru Ota, et al., proposes a unmanned aerial vehicles topology discovery scheme to discover the physical topology with low-cost and high accuracy. The authors use vehicles as mobile anchors and the cloud platform to analyze the received information to determine undiscovered physical topology.

The forth paper, "Energy Efficient Mode Selection, Base Station Selection and Resource Allocation Algorithm in D2D Heterogeneous Networks" by Zhufang Kuang, Gongqiang Li, et al., studies the joint problem of DUEs mode selection, base station selection, channel allocation and power allocation in D2D communications, with the objective to optimize the energy efficiency of the system.

The fifth paper, "Revenue-Optimal Task Scheduling and Resource Management for IoT Batch Jobs in Mobile Edge Computing" by Jiwei Huang, Songyuan Li, et al., focuses on the problems of dynamic task scheduling and resource management in mobile edge computing systems, with the specific objective of achieving the optimal revenue earned by edge service providers.

The sixth paper, "A Trust Evaluation System Based on Reputation Data in Mobile Edge Computing Network" by Xiaoheng Deng, Jin Liu, et al., addresses security issues to facilitate mobile edge computing. The authors propose a three-tier trust evaluation framework to ensure that the edge nodes in the network are qualified, capable, and reliable.

The seventh paper, "A Near-optimal Content Placement in D2D Underlaid Cellular Networks" by Guangsheng Feng, Yue Wang, et al., investigates the content placement problem to maximize the cache hit rate in Device-to-Device underlaid cellular networks. The authors prove that the problem under consideration is a submodular function maximization problem. Then the authors propose an algorithm with approximation guarantee. 
The eighth paper, "Data Driven Hybrid Edge Computingbased Hierarchical Task Guidance for Efficient Maritime Escorting with Multiple Unmanned Surface Vehicles" by Jiajia Xie, Jun Luo, et al., proposes a hierarchical scheme for unmanned surface vehicles to guard a valuable ship. The problem falls into the scope of sparse rewards and long-time horizon which can be addressed by multi-agenet reinforcement learning schemes.

The ninth paper, "Polynomial Regressors Based DataDriven Control for Autonomous.

Underwater Vehicles" Hongran Li, Weiwei Xu, et al., proposes a data-driven control approach based on polynomial regressors for autonomous underwater vehicles.

The tenth paper, "Performance Analysis of Edge-PLCs enabled Industrial Internet of Things" by Yanjun Peng, Peng Liu, et al., conducts performance analysis of different partition instances of the memory in the edge-programmable logic controllers by modeling the problem as a single server queueing system.

The eleventh paper, "Content Caching Based on Mobility Prediction and Joint User Prefetch in Mobile Edge Networks" by Genghua Yu, Zhigang Chen, et al., proposes a content caching strategy based on mobility prediction and content prefetching. By enabling user to prefetch data from the edge cache devices, the backhaul load can be significantly reduced.

To conclude this editorial, we would like to thank Prof. Xuemin Shen for all the advice regarding this special issue, as well as Ms. Katherine Moretti for the help during the publication process. Furthermore, we would like thank all the contributing authors and the reviewers, without whom we would not have this special issue.

Publisher's note Springer Nature remains neutral with regard to jurisdictional claims in published maps and institutional affiliations. 